\title{
ASSISTANCE SYSTEM FOR AN AUTOMATED LOG-QUALITY AND ASSORTMENT ESTIMATION BASED ON DATA-DRIVEN APPROACHES USING HYDRAULIC SIGNALS OF FORESTRY MACHINES
}

\author{
Chris Geiger*, Niklas Maier, Florian Kalinke, Marcus Geimer \\ Institute of Mobile Machines, Karslruhe Institut of Technology, Rintheimer Querallee 2, 76131 Karlsruhe \\ * Corresponding author: Tel.: +49 721 608-48642; E-mail address: chris.geiger@kit.edu
}

\begin{abstract}
The correct classification of a logs assortment is crucial for the economic output within a fully mechanized timber harvest. This task is especially for unexperienced but also for professional machine operators mentally demanding. This paper presents a method towards an assistance system for machine operators for an automated log quality and assortment estimation. Therefore, machine vision methods for object detection are combined with machine learning approaches for estimating the logs weight based on a Convolutional Neural Network (CNN). Based on the dimensions of the object 'log', a first categorisation into a specific assortment is done. By comparing the theoretical weight of a healthy log of such dimensions to the real weight estimated by the $\mathrm{CNN}$-based crane scale, quality reducing properties such as beetle infestation or red rod can be detected. In such cases, the assistance system displays a visual warning to the operator to check the loaded log.
\end{abstract}

Keywords: Assistance System, Log Assortment, Crane Scale, Machine Learning, Machine Vision, Forwarder, Convolutional Neural Network

\section{MOTIVATION}

In a fully mechanized timber harvest, a harvester fells the trees and cuts them into logs of specific length according to the log quality. Afterwards, a forwarder loads the logs and moves them from the logging area to a forest road. Here, the operator piles the logs into different assortments regarding their specifications such as length and quality. Loading and piling sums up to $85 \%$ of total working hours. [1-4]

It requires experienced operators with a background in forestry to fulfil these tasks efficiently, economically and sustainably. The main challenges forestry service companies currently face are a high employee fluctuation and a simultaneously increasing average age of the machine operators $[5,6]$. Training of a new operator takes 9 months on average [7], resulting in reduced productivity during this time. Hence, this lack of experienced operators results in an economical deficit within the forestry sector.

\section{ASSISTANCE SYSTEM FOR ASSORTMENT ESTIMATION}

Especially new, young operators show a lack of experience in estimating the log dimension and quality during loading due to an insufficient background in forestry. A wrong assortment assignment leads to a quality reduction of the whole pile where the log was placed and reduces the overall profit of the forest owner and consecutively the forestry contractor. The log quality is based on the log's health and decreases with defects, e.g. red rot or bark beetle infestation at spruces, resulting in reduced weight compared to a healthy log. The aim of this paper is to close this gap in experience by presenting a method for an automated $\log$ quality and assortment estimation during a continuous loading cycle, exemplary shown for spruces. Figure 1 displays the functional principle of the assistance system, which combines two parallel branches, one for measuring the weight and one for measuring the $\log$ dimensions, into an assortment estimation for forestry machine operators. The bottom branch receives the weight of the log via a $\mathrm{CNN}$, mainly 

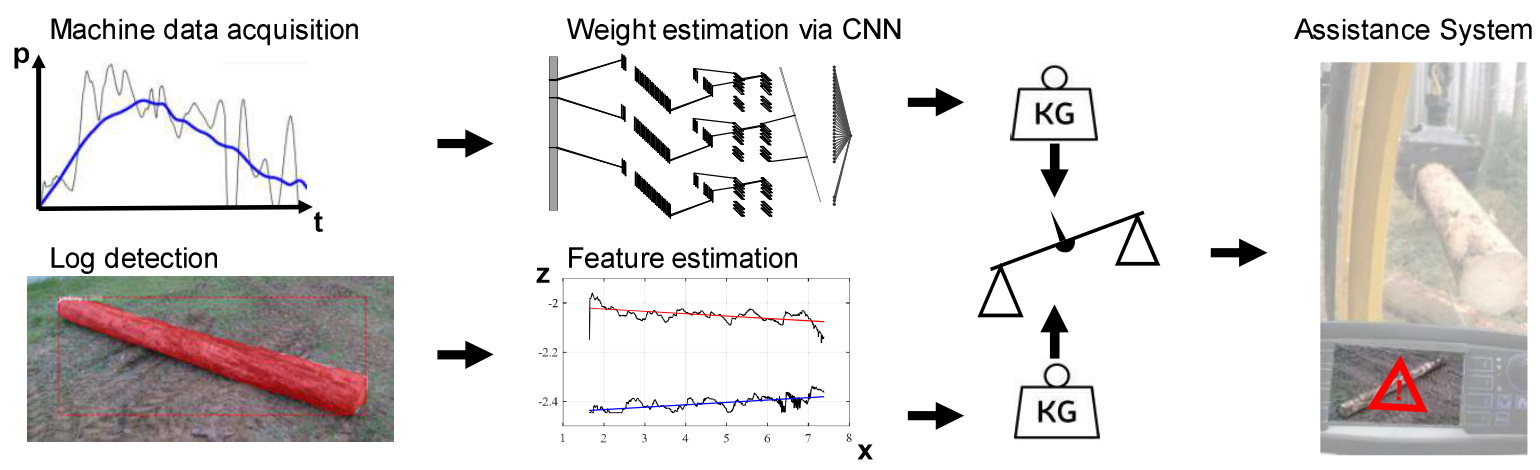

Figure 1: Flow Chart of the Assistance System

based on the input of the hydraulic pressure of the inner boom cylinder and the grapple position. The upper branch estimates the $\log$ diameter and length via object detection methods, resulting in the log volume. Assuming the density of a healthy $\log$, the theoretical weight of the $\log$ is calculated and compared in the next step to the measured weight. In cases of significant differences, e.g. if the log is lighter due to red rot, a warning appears at the machine operators display, cf. Figure 1 right. In the following, Chapter 3 shows the state of the art of object detection and crane scales. The two branches for the assistance system are explained in detail in Chapter 4 for the object detection and in Chapter 5 for the databased crane scale.

\section{STATE OF THE ART}

Currently, a system for forwarders estimating the $\log$ assortment automatically is unknown to the authors. Nevertheless, different approaches for the necessary subsystems of such an assistance system, especially measurement systems for the weight and dimensions of a log, are investigated by industry and research.

\subsection{Object Detection of Logs}

Machine vision is an essential part of many current research projects in the field of automation. However, research focusing on automatic recognition of logs are quite rare. [8] deals with general object recognition in point clouds. The authors discuss a robust method to fit cylinder models in incomplete point cloud data. Based on laser scanning, a method is proposed that combines Principal Component Analysis and RANSAC algorithms [ibid].
A forestry related research topic is the development of an algorithm for recognition and pose estimation of logs using a 3D structured light camera [9]. Herein, a method for analyzing the shape of a log by segmentation and surface patching is described. The focus is on the detection of rotational symmetries in point clouds. With this method, the determination of the volume and location of logs is implementable in automated processes, limited with a success rate under $33 \%$ at a distance of $5 \mathrm{~m}$. [9]

In order to evaluate object recognition algorithms, it is important to deal with the deviation between the determined values and the actual values. In [10] the authors focus on research in the field of volume measurements of logs. The main objective is the experimental determination of the actual log volume according to the Archimedean principle. Comparing the resulting volume data to the harvester measuring system as well as the in forestry common manual method shows an absolute volume error range up to $27 \%$ for the $5 \%$ - $95 \%$ quantiles. [10]

\subsection{Crane Scales}

Commercial available crane scales for weighing the grapple content are provided by forestry machine manufactures $[11,12]$. These scales are mounted between grapple and telescope, resulting in worse maneuverability due to negatively changed crane kinematics. Furthermore, measuring with high accuracy requires a static crane state $[13,14]$, resulting in reduced overall productivity.

A crane scale based on artificial neural networks was first presented by the authors in [15] and [16]. Based on a Long Short-Term Memory (LSTM) architecture, an Artificial Neural Network (ANN) weighs the grapple 
content within a continuous loading process. The focus of this method is on an estimation of the logged mass of a working day in post-processing procedure. During testing, the ANN shows for single loading cycles an average full scale error of $1.5 \%$ per $1000 \mathrm{~kg}$, resulting in an example loading scenario in a total mass error of $1.2 \%$ for a fully loaded forwarder with 10 tons payload [16].

\section{LOG DETECTION ESTIMATION}

AND SHAPE

The aim of the proposed recognition algorithm is the automated determination of $\log$ volumes. The approach combines object recognition in 2D images and 3D point clouds. Using the feature of ordered point clouds allows assigning a space point to the corresponding pixel in a twodimensional image and vice versa.

\subsection{Camera technology}

An Intel ${ }^{\circledR}$ RealSense ${ }^{\mathrm{TM}}$ D415 stereo camera is used for the object recognition method. The camera functions are based on active stereoscopy technology. The manufacturer specifies an application range of $0.45 \mathrm{~m}$ to $10 \mathrm{~m}$ for depth detection. The D415 generates point clouds via the coupling of two infrared cameras, an active infrared projector and a RGB camera. [17]

A defined number of space points, represented by a location matrix $\underline{M}$ and a corresponding RGB colour matrix $\underline{\mathrm{C}}$, are orderly recorded.

$$
\underline{M}=\left(\begin{array}{ccc}
x_{1} & y_{1} & z_{1} \\
\vdots & \vdots & \vdots \\
x_{i} & y_{i} & z_{i}
\end{array}\right) \quad \underline{C}=\left(\begin{array}{ccc}
r_{1} & g_{1} & b_{1} \\
\vdots & \vdots & \vdots \\
r_{i} & g_{i} & b_{i}
\end{array}\right)
$$

Therefore, the properties of a point with an index $i$ are defined by six scalar quantities:

$$
\text { point }_{i}=\operatorname{object}\left(x_{i}, y_{i}, z_{i}, r_{i}, b_{i}, g_{i}\right)
$$

Using the Intel ${ }^{\circledR}$ RealSense ${ }^{\text {TM }}$ SDK 2.0, the depth

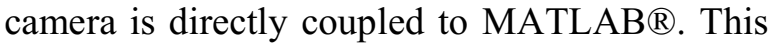
enables the integration of recordings into automated processes. Another advantage of the used camera is the comparatively low purchase price, which allows a cost-effective testing of the proposed method.

\subsection{Data acquisition}

Due to challenging environmental conditions in forestry, the camera was mounted on the forwarders cabin roof. Therefore, the recorded point clouds lie at a defined angle in space. This setup was considered when recording the validation and verification data. Varying mounting angles and heights improves the robustness of the method due to the independence from the installation setting. The resolution of the camera was set to $1280 \times 720$ pixels, which outputs a point cloud with 921.600 points.

\subsection{Method of log recognition}

Because the elements of point clouds are ordered, an RGB image can be generated from the recorded colour matrix $\underline{C}$. Each described colour value of a point is assigned to a pixel in the $2 \mathrm{D}$ image via the indices of the point cloud.

Using the ordering of the point cloud together with the recorded color matrix $\mathbf{C}$ as lookup-table, an RGB image $I \in \mathbb{R}^{1024 \times 1024 \times 3}$ with pixels $I_{j k}$ can be generated. Next, $\mathbf{I}$ is processed by a Mask R-CNN [18], which we adapt for log recognition by transfer learning [19]. The network outputs bit-masks for each detected log, which map all $I_{j k}$ to the corresponding object, cf. Figure 1 left. For the Transfer Learning 100 hand-annotated pictures of single logs were used. The training set consisted of 80 pictures, with the remaining 20 used for validation. An accuracy for log detection of $100 \%$ was achieved.

The object pixels, corresponding to the bitmask, are recalculated into the three-dimensional space. Thus, the $3 \mathrm{D}$ points are assigned to the object ' $\log$ '. The volume calculation is based on the recognized log length and the local log diameter.

The location matrix of the point cloud is rotated for further processing of the data via matrix transformation. The aim is to rotate the location matrix in a way that the log lies on the $\mathrm{X}-\mathrm{Y}$ plane parallel to the $\mathrm{Y}$-axis. Thus, the Zcoordinates of the location matrix $\underline{M}$ describe the local $\log$ diameters as a height profile, the Xcoordinates the log width and the Y-coordinates the log length. As a reference for the necessary rotations, an M-estimator Sample Consensus (MSAC) algorithm is used to fit a plane to the point cloud data [20], which represents the ground. With the normal vector of the plane, the rotation angles around the $\mathrm{X}$ - and $\mathrm{Y}$-axis are determined. For the rotation on the Z-axis the direction of the log must be determined. 


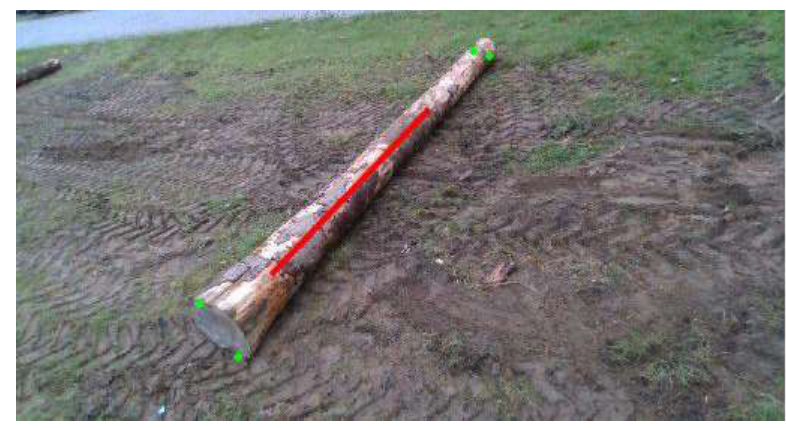

Figure 2: RGB data with determined log direction

An edge detection of the outline 2D image from the Mask R-CNN is carried out. The outermost points of the log are determined (top, bottom, right and left), cf. Figure 2 in green. An imaginary box is created around the log based on the four boundary points. The two centres of the front and rear boundary points are calculated. The $\log$ direction is determined by connecting these two centres. In order to ensure that the described points are on the logs surface, the points are moved along the direction axis. Figure 2 shows the RGB image with the generated direction vector in red as a result of the edge detection. The result of the transformation of the location matrix is shown in Figure 3, where the log lies parallel to the X-Y-Plane of the original system. After the data has been defined in the original space, the $\log$ length is determined. For the further process, the set of points is reduced to the pure log points that the Mask R-CNN generated as output.

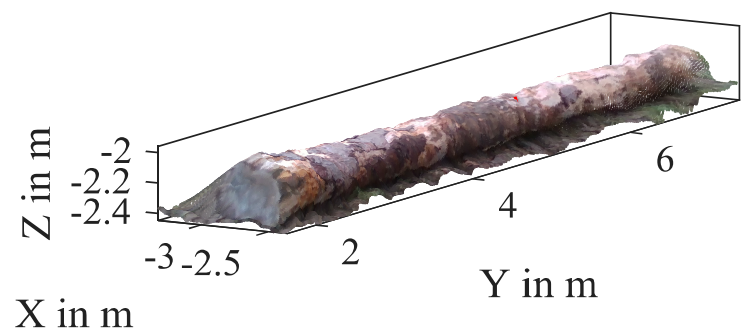

Figure 3: Rotated point cloud with indicated log direction (red)

\subsection{Shape estimation}

The local minima and maxima of the Zcoordinates along the $\mathrm{Y}$-direction are determined. A profile of the top and bottom points is created as a matrix of Y-and Z-coordinates. To estimate the log length, only the profile of the top side is analysed. By using the MSAC algorithm, the points of the Y-Z-location matrix are fitted as a straight line that approximates the course of the diameter over the log length. We define the end of the log as the last point of intersection between the Y-Z curve and the approximated diameter curve from the MSAC algorithm. The same algorithm is used for the bottom side of the log. Figure 4 shows the results of the fitting process.

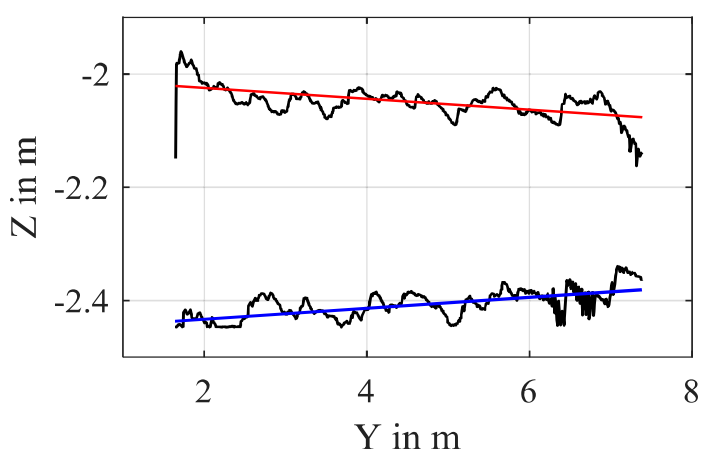

Figure 4: Point data describing log diameter and length with fitted lines

\subsection{Error Estimation}

Due to technical limitations of the camera, errors occur when measuring the depth. The error is estimated using the manufacturer's formula of the root-mean-square deviation. [21]

$$
\text { Depth RMS error }=\frac{\text { Distance }^{2} * \text { Subpixel }}{\text { focal length } * \text { Baseline }}
$$

With the used camera settings and an estimated maximal working distance of $8 \mathrm{~m}$, a root-meansquare deviation of the depth of $93 \mathrm{~mm}$ is calculated. The error increases quadratically with the distance and is influencing the length and diameter determination. Especially the latter leads to an error in the volume calculation.

\subsection{Verification Data}

To verify and validate the functionality of the $\log$ detection and shape estimation, 11 logs with a length about $5.2 \mathrm{~m}$ and a diameter range of $0.3 \mathrm{~m}$ to $0.5 \mathrm{~m}$ were tested. Figure 5 shows the errors of the diameter and length determination, as well as the error of the calculated volume for these logs. As the absolute error in length is less than $7 \%$ with a median of $-0.4 \%$, the diameter estimation shows an error range between $-11 \%$ to $18 \%$ with a median of $-5.9 \%$. Here, the camera error described in Chapter 4.5 leads to a significant deviation of the measurement results. This diameter error influences the volume error significantly as it is included quadratically in the volume calculation. 


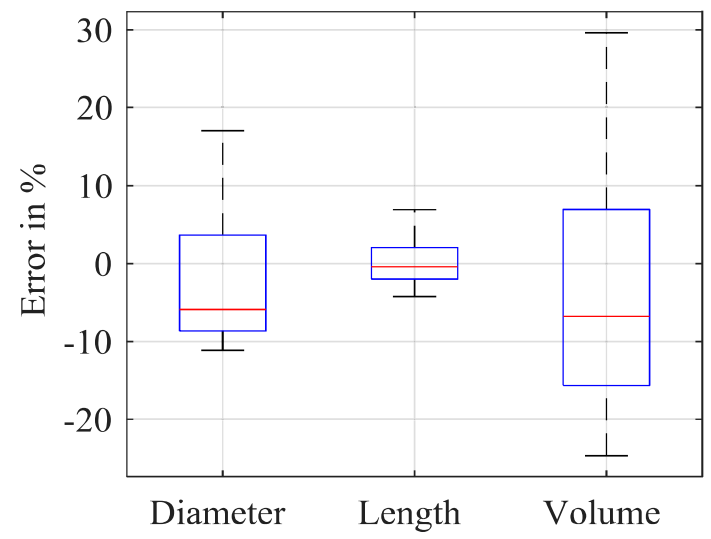

Figure 5: Errors of diameter, length and volume determination

As a basis for the explanation of the assistance system, the best results are presented in the following. With these results, shown in Table 1, the coupling possibilities of the crane scale with the $\log$ recognition algorithm will be demonstrated exemplarily in chapter 6 . The real volume of the $\log$ presented in this example is $\mathrm{V}=0.55 \mathrm{~m}^{3}$, whereby the calculated volume determined by our method is $\mathrm{V}=0.5448 \mathrm{~m}^{3}$.

Table 1: Results for log with least error

\begin{tabular}{ll}
\hline Log parameter & Error [\%] \\
\hline Diameter & 2.88 \\
Length & -2.73 \\
Volume & 1.26 \\
\hline
\end{tabular}

The results show that the accuracy of the proposed method depends primarily on the camera, cf. Chapter 4.5. In addition, we verified this dependency by using a logs point cloud achieved within the simulation environment GAZEBO, resulting in a volume error of $0.44 \%$. In future implementations of this system, it will be necessary to use a depth camera with a lower depth resolution error to show the benefit of the presented method for the assistance system.

\section{CRANE SCALE BASED ON A CONVOLUTIONAL NEURAL NETWORK (CNN)}

Beneath remaining constant crane kinematics, the objective of a data-based crane scale is, that modelling the crane with its non-linearity's and unknown parameters like friction coefficients is not necessary. Therefore, the function drive of the machine is mapped to an artificial neural network [16].
In a static situation, the mass of the grapple content can be calculated, neglecting adhesive friction, on the equilibrium of moments based on the pressure of the inner boom cylinder (IBC) and the grapple distance, cf. Figure 6. Avoiding a reduced productivity during loading processes due to stoppage for a weight measurement, the ANN represents a dynamic model of the crane. Therefore, the weighing process can be executed automatically during continuous loading processes.

In contrary to the in [16] presented approach of a crane scale based on a LSTM architecture, a convolution-only architecture is presented in this paper. Hereby, this architecture avoids drawbacks of Recurrent Neural Networks (RNN) with LSTM cells such as higher training and testing time, parallelization issues as well as higher sensitivity concerning overfitting or first examples seen [22]. Based on the idea of [22, 23] and partially conducted by [24], a Convolutional Neural Network was developed to fulfil the task of estimating the mass of the grapple content.

Outer boom

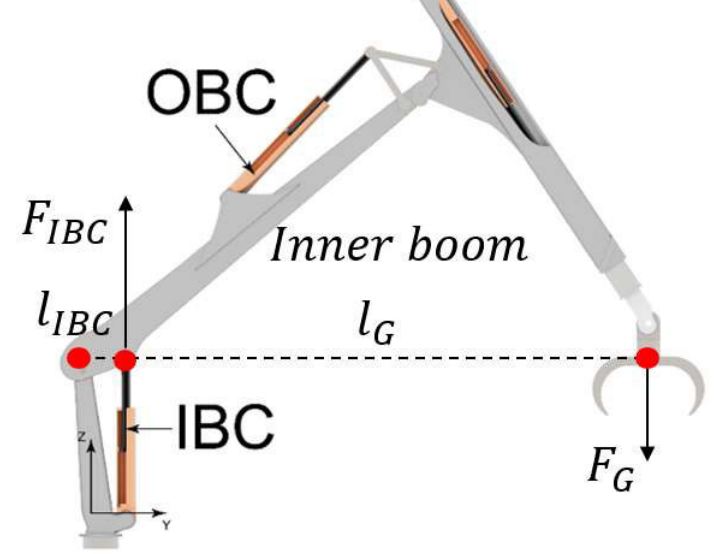

Figure 6: Forestry Crane [16]

\subsection{Data Pre-Processing}

The challenge of achieving a robust, real-world applicable setup for a crane scale at forestry conditions is using the least sensors possible. In modern in-market forestry cranes, each joint is equipped as standard with a kinematic sensor. With the presented setup, we only added one hydraulic pressure sensor to the system measuring the pressure of the inner boom cylinder (IBC). With this principle, the crane scale is independent regarding the used hydraulic systems for the crane drive [25] as well as the 
machine manufacturer. Furthermore, the operator's input in form of the joystick signals, which are just like the other sensors readable from the machine's CAN-Bus, is used as input signals for the $\mathrm{CNN}$ [16]. The hydraulic signal of the pressure of the IBC is used as a direct as well as a preprocessed input into the network. Therefore, the pressure signal is filtered by means of a Savitzky-Golay filter. Furthermore, the pressures frequency spectrum calculated by a fast Fourier transform serves as an additional input. Finally, the oil temperature in tank, always available for monitoring and safety purposes on forestry machines, is an input signal. The grapple position as input results from processing the kinematic sensor of each crane joint, the grapple velocity by differentiating the position. [16]

The input consists of time-series data. In a first step, the input signals are standardized. Afterwards, the standardized signals are normalized between values of 0 and 255 and saved as an greyscale image [24]. The resulting image has the size of $18 \times 250$ pixels corresponding to the 18 input signals and 250 time steps, meaning that each pixel is equivalent to a specific measurement value.

\subsection{Architecture of the CNN}

For the CNN architecture, we use three parallel branches, each consisting of Convolutional Layers, Rectified Linear Units (ReLU) Layers and Pooling Layers [22, 24]. Figure 7 visualizes exemplary the first branch. As input for the CNN serve the converted time series data as an $18 \mathrm{x}$ 250 pixel greyscale image.

The first Convolutional Layer consists of 128 filters with a filter size of $18 \times 1$ and stride one. Hence, this filter covers all input signals at each time step. Directly connected to the
Convolutional Layer is the ReLU activation function $f(x)=\{(x, x \geq 0 ; 0, x<0)$, which passes only input values greater and equal to zero [26]. Subsequently, the Max Pooling Layer with a size of $5 \times 1$ and stride 5 downsamples the input from $250 \times 1$ to $50 \times 1$. This corresponds to using only the maximum value of the filtered 18 input signals in a time range of five time steps. The second Convolutional Layer consists of 32 filters with a filter size of $1 \times 1$ and stride 1 . As input serve the 128 channels resulting from the previous layers. Therefore, this recombines the previously divided input signal. The second Max Pooling Layer condense the input to $25 \times 1$, whereby the Concatenation Layer receives only 800 inputs for this branch.

The first Convolutional Layer of the second branch consists of 128 filters with a filter size of $18 \times 5$ and stride five. Therefore, already with the first layer in the $\mathrm{CNN}$, five time steps are considered. In contrary to the first branch, no max pooling takes place between the first and second Convolutional Layer. The same principle is also applied for the third branch, expect for the fact that the filter size here is $18 \times 51$ with stride 4 , covering therefore a wider time range. For both second and third branch, the second Convolutional Layer and Max Pooling Layer is the same as in the first branch, resulting to 800 input values for each branch for the Concatenation Layer. The Concatenation Layer combines the outputs of each branch and transfers the values to the Fully Connected Layer. Therefore, only 2400 weights have to be determined during training for this layer. The last layer is a regression output layer, meaning that the output of the $18 \times 250$ image is a single integer value representing the mass of the grapple content.
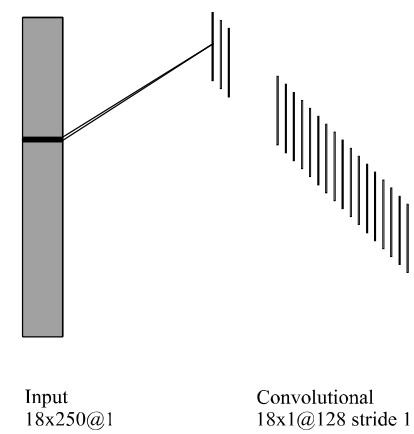

Input
$18 \times 250 @ 1$ 18x1@128 stride 1

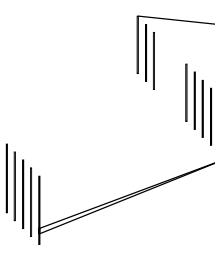
$\begin{array}{ll}\text { MaxPooling } & \text { Convolutional } \\ \text { 5x1@128 stride 5 } & \text { 1x1@32 stride1 }\end{array}$ 1x1@32 stride1

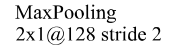

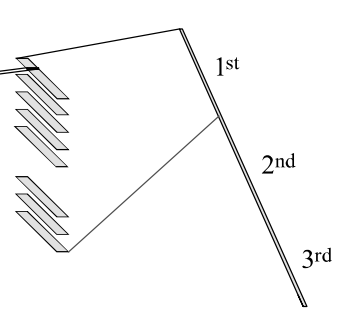

Concatenation

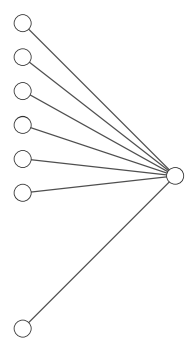

FullyConnected

Figure 7: First Branch of used Convolutional Neural Network 


\subsection{Training of the $\mathbf{C N N}$}

We logged training data for the $\mathrm{CNN}$ by recording 550 loading cycles under reproducible laboratory conditions between August 2018 and March 2019, while the forwarder as well as the logs were placed on a flat surface. Three different machine operators with, from beginner to professional, varying expert level performed the loading cycles. The real log mass was determined by a scale with $0.5 \mathrm{~kg}$ accuracy. The log lengths were about $5.2 \mathrm{~m}$, the log masses were in a range of 100 to $600 \mathrm{~kg}$ with a varying diameter up to $50 \mathrm{~cm} .[16]$

31 loading cycles were reserved for testing of the crane scale. The remaining 519 cycles were split during training to $90 \%$ for training and $10 \%$ for validation. Due to the small amount of training data with its resultant issues [27], we used a six-fold cross validation, meaning that all 519 training cycles were randomly separated six times into a training data set and a validation data set [24].

For training, we set the learning rate to 0.01 , the mini batch size to 20 and used a maximum of 30 epochs. Each training consists of 20 iterations, resulting overall in 120 networks due to the 6 -fold cross validation. After the first training, we successively performed two further training passes with a learning rate of 0.001 using the weights of the pre-trained networks.

\subsection{Results of Training and Validation Data Set}

We evaluate the performance of the $\mathrm{CNN}$ on the trainings and validation data set by the percentage error resulting from difference of the predicted mass to the real mass divided by the real mass. Figure 8 shows the gauss distribution of the Prediction Error (PE) for the validation set (left) and the training set (right) in a range of $-40 \%$ to $40 \%$. The training data set shows, based on 56,160 values, a mean PE of $0.23 \%$ and a median PE of $-0.0263 \%$ with a standard deviation of $4.67 \%$. The validation data set displays, based on 6,120 values, a mean PE of $0.46 \%$ and a median PE of $-0.0095 \%$ with a standard deviation of $7.33 \%$.

Table 2 lists the Prediction Error for both training and validation data with its $5 \%, 25 \%$, $75 \%$ and $95 \%$ quantiles. Taking the $5 \%$ and $95 \%$ quantiles as well as the mean $\mathrm{PE}$, into account, the CNN shows a slight tendency of overestimating the mass with its prediction. Consecutively, $50 \%$ of the predicted masses have an absolute PE less than $1.71 \%$ (training data set) respectively less than $3.13 \%$ (validation data set).
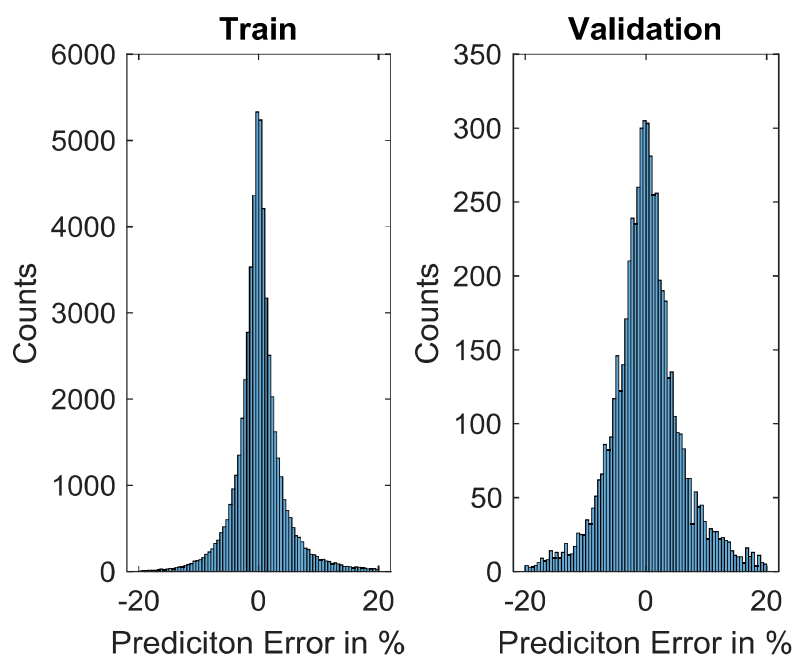

Figure 8: Prediction Error Distribution

Therefore, we observe no significant overfitting of the CNN, resulting in a robust network for detecting the mass based on a minimal amount of sensor data.

Table 2: Statistical analysis of the CNN

\begin{tabular}{lll}
\hline Quantile [\%] & $\begin{array}{l}\text { Prediction Error } \\
\text { in Training Data } \\
\text { Set [\%] }\end{array}$ & $\begin{array}{l}\text { Prediction Error } \\
\text { in validation Data } \\
\text { Set [\%] }\end{array}$ \\
\hline 5 & -6.25 & -9.46 \\
25 & -1.69 & -2.95 \\
75 & 1.71 & 3.13 \\
95 & 7.44 & 12.18 \\
\hline
\end{tabular}

\subsection{Results on Test Data Set}

Reducing the variance of the mass prediction, we use bagging for testing [28]. Therefore, we determined the $20 \mathrm{CNNs}$ with the least mean PE on the validation data set. During testing, by using a trimmed mean, the mass of the grapple content is averaged based on these networks without the upper and lower $10 \%$ of the predictions. Embedding this evaluation method into the assistance system increases the robustness of the same against varying machine operators and environmental factors.

The test data set consists of the 11 loading cycles for the in Chapter 4 evaluated logs and 20 randomly chosen loading cycles. None of these 
cycles was used during training or testing of the CNN. The loading cycles were performed by two different machine operators with different experience level, one advanced beginner and one professional. In total, these cycles sum up to $6888 \mathrm{~kg}$, tallying nearly to a complete payload of a forwarder. The estimated mass by the $\mathrm{CNN}$ for all cycles is in total $6873 \mathrm{~kg}$, resulting in an error of $-0.22 \%$. Figure 9 shows the prediction error of the validation set. The Prediction Error of single $\operatorname{logs}$ is in a range of $-7.73 \%$ to $10.73 \%$ with a mean PE of $1.20 \%$.

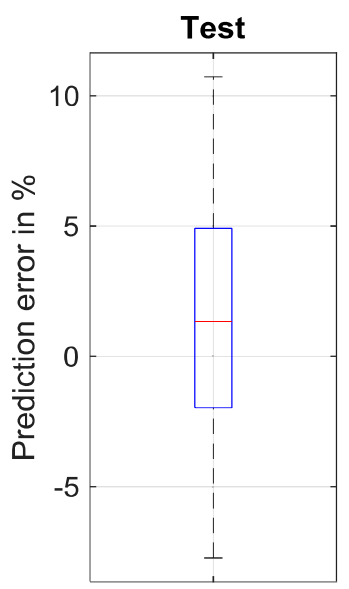

Figure 9: Test Data set

The range of the PE in the validation data set corresponds very well to the error range in the test data set. This underpins the stability and robustness of the Convolutional Neural Network. The fact, that the absolute error can be higher than $10 \%$ is no critical argument against the shown method and network architecture as only a minimum of data was used for training. Increasing training data will reduce the error range significantly.

\section{ASSISTANCE SYSTEM}

The in Figure 1 visualized assistance systems for $\log$ quality and assortment estimation is the combination of both $\log$ detection and mass prediction shown in the previously chapters. By using the log with the best volume estimation, the method and functionality of the assistance system is explained in the following. In this exemplary case, the evaluated log shows a significant bark beetle infestation.

In the first step, the length and diameter of the $\log$ specifies its assigned assortment. In the example case, the $\log$ is appointed to the assortment FL (log with standard length) and a diameter class D3b due to a diameter of $0.36 \mathrm{~m}$ [29]. In the second step, the mass of the log is calculated based on the log volume and a green wood density of $906 \mathrm{~kg} / \mathrm{m}^{3}$, assuming that the $\log$ is of healthy state without defects. In our example, this results in a theoretical log weight of $494 \mathrm{~kg}$. Afterwards, this value is compared to the mass predicted by the Convolutional Neural Network, cf. Figure 1. As this mass has a predicted value of $404 \mathrm{~kg}$ - with a PE of $6.60 \%$ the $\log$ weighs only $81.78 \%$ of the expected value calculated with the green wood density. The deviation is significantly higher than the absolute PE of the Test Data Sets $5 \%$ and $95 \%$ quantiles, resulting in a low certainty of an outlier. The threshold for sending a visual warning to the operator is when the mass difference estimated by the volume information and the CNN output is greater than $15 \%$. This corresponds directly to the quality classification, as with a red rot percentage over $15 \%$ the log quality decreases [29].

By displaying this warning and the log information on the machine operators monitor, he is able to double-check the log quality. As further information, the theoretical mass for beetle infested $\log$ s with $373 \mathrm{~kg}$ is displayed, which varies about $7.76 \%$ from the $\mathrm{CNN}$-estimated mass and $1.67 \%$ from the real mass. Therefore, if there is no visual sign of red rot at the front faces of the $\log$, the operator defines the $\log$ to quality D due to beetle infestation [29].

\section{CONCLUSION}

The right assignment of $\operatorname{logs}$ to their specific assortment is crucial for an economical harvesting process. The presented method describes an assistance system for machine operators, which supports them in the task of the assortment estimation.

The assistance system combines two parallel branches of object and weight detection to estimate the logs dimension and quality, resulting in its assortment. The functionality of the presented method was verified within this paper.

For object detection, an Intel RealSense D415 depth camera was used. Due to a root mean square error up to $0.1 \mathrm{~m}$ within working distance, the logs diameter estimation shows an absolute error range of $17 \%$, resulting in an error range from $-25 \%$ to $30 \%$ regarding the volume 
calculation. Therefore, the chosen depth camera limits the potential of the assistant system. The volume error influences directly the theoretical mass calculation based on the green wood density for logs of healthy state. This error could be reduce significantly with an industry-standard depth camera. Nevertheless, the approach for detecting a log via machine learning methods and the consecutive dimension estimation could be verified under laboratory conditions. Of special use is the log length estimation for machine operators, as this parameter defines the assortment of the $\log$ in the first place. Distinguishing the common log lengths of $3.6 \mathrm{~m}$, 4.0 and 5.0 is difficult in real forestry conditions and mentally demanding.

For the weight detection system, a databased approach resting on a Convolutional Neural was developed and successfully tested. The chosen $\mathrm{CNN}$ shows a prediction error for single loading cycles between $-2 \%$ to $5 \%$ for the $25-75 \%$ quantiles of the test data set. This corresponds to a full scale error of $2 \%$ with a maximum absolute error of $20 \mathrm{~kg}$ in this range. Due to the results at the validation data set, the CNN shows a high robustness against varying machine operators and changing environmental conditions. Furthermore, the $\mathrm{CNN}$ achieves a certain degree of abstraction as in the period of data recording parameter settings and optimizations of the hydraulic system were done. Therefore, it is possible to transfer the crane scale to other machines with the same crane type, accomplishing a universal usage of the presented method.

\section{OUTLOOK}

In a first step, accuracy of the log dimension estimation will be significantly increased using an industry standard depth camera. The log detection will be extended from the twodimensional image to an object detection in a 3D dataset. Furthermore, the algorithms for this estimation will be improved towards fitting a three-dimensional truncated cone in the point cloud data which approximates the $\log$. The combination of multiple point clouds recording the same scenery towards one, holistic point cloud with a high accuracy will be investigated.

For the crane scale, additional training cycles under varying conditions will be recorded in forestry environments. Therefore, the accuracy and robustness of the presented method will improve significantly. After a further optimization of the algorithms, the assistance system will be implemented as a near-time, online system in the forwarder cabin.

\section{REFERENCES}

1. Cacot E, Bigot M, Cuchet E (2006) Developing full-mechanized harvesting systems for broadleaved trees: a challenge to face the reduction of the manual workforce and to sustain the supply of hardwood industries. In: 2006 Council on Forest Engineering (COFE) Conference Proceedings: "Working Globally Sharing Forest Engineering Challenges and Technologies Aroun

2. Nurminen T, Korpunen H, Uusitalo J (2006) Time consumption analysis of the mechanized cut-to-length harvesting system. Silva Fenn. 40(2). doi: 10.14214/sf.346

3. Kuptz D, Turowski P, Hartmann H et al. (2015) Optimale Bereitstellungsverfahren für Holzhackschnitzel

4. Manner J, Palmroth L, Nordfjell T et al. (2016) Load level forwarding work element analysis based on automatic follow-up data. Silva Fenn. 50(3). doi: 10.14214/sf.1546

5. Schulz C (2017) Berufspolitisches Engagement gefragt!: Interview von Christoph Schulz mit Christian Feußner, beide Angestelltenvertreter im Gesamtpersonalrat von Hessen-Forst. BDF aktuell 58(1): 20-21

6. Wippel B, Viergutz M (2014) Stärkung der Kooperations-strukturen von Forstunternehmern in Baden-Württemberg. Abschlussbericht

7. Purfürst T (2009) Der Einfluss des Menschen auf die Leistung von Harvestersystemen. Dissertation, Technische Universität Dresden

8. Nurunnabi A, Sadahiro Y, Lindenbergh R (2017) ROBUST CYLINDER FITTING IN THREEDIMENSIONAL POINT CLOUD DATA. Int. Arch. Photogramm. Remote Sens. Spatial Inf. Sci. XLII-1/W1: 63-70. doi: 10.5194/isprsarchives-XLII-1-W1-63-2017

9. Park Y, Shiriaev A, Westerberg S et al. (2011 2011) 3D log recognition and pose estimation for robotic forestry machine. In: 2011 IEEE International Conference on Robotics and Automation. IEEE, pp 5323-5328

10. Hohmann F, Ligocki A, Frerichs L (2017) HARVESTER MEASURING SYSTEM FOR 
TRUNK VOLUME DETERMINATION: COMPARISON WITH THE REAL TRUNK VOLUME AND APPLICABILITY IN THE FOREST INDUSTRY. Bulletin of the Transilvania University of Brasov

11. Komatsu Options Forwarders. https://www.komatsuforest.com/forestmachines/our-forwarders/forwarder-options. Accessed 13 Apr 2019

12. Ponsse PONSSE ATTACHMENTS ADD VALUE TO YOUR MACHINE. http://www.toimilgruas.com/pdf/Attachment_E NGL.pdf. Accessed 13 Apr 2019

13. Korten S, Kaul C (2012) Optimierung der Transportprozesse bei Holzernte und Rundholztransport durch den Einsatz von Wechselbrücken: Schlussbericht

14. Wide MI (2012) Väg rätt redan i skogen. SKOGFORSK

15. Geiger C, Starke M, Greff D et al. (2018) THE POTENTIAL OF A WEIGHT DETECTION SYSTEM FOR FORWARDERS USING AN ARTIFICIAL NEURAL NETWORK. In: FORMEC 2018 - Improved Forest Mechanisation: mobilizing natural resources and preventing wildfires

16. Geiger C, Greff D, Starke $M$ et al. Entwicklung und Evaluation eines Wiegesystems für Forstkräne auf Basis von künstlichen neuronalen Netzen. LANDTECHNIK, Bd. 74 Nr. 5 (2019)

17. Giancola S, Valenti M, Sala R (2018) A Survey on 3D Cameras: Metrological Comparison of Time-of-Flight, Structured-Light and Active Stereoscopy Technologies. Springer International Publishing, Cham

18. He K, Gkioxari G, Dollar P et al. (2017) Mask R-CNN. In: The IEEE International Conference on Computer Vision (ICCV)

19. Waleed A (2017) Mask R-CNN for object detection and instance segmentation on Keras and TensorFlow. https://github.com/matterport/Mask_RCNN. Accessed 25 Nov 2019

20. Torr PHS, Zisserman A (2000) MLESAC: A New Robust Estimator with Application to Estimating Image Geometry. Computer Vision and Image Understanding 78(1): 138-156. doi: 10.1006/cviu.1999.0832

21. Grunnet-jepsen A, Sweetser JN, Woodfill J BestKnown-Methods for Tuning Intel ${ }^{\circledR}$ RealSense ${ }^{\mathrm{TM}}$ D400 Depth Cameras for Best Performance. https://dev.intelrealsense.com/docs/tuning- depth-cameras-for-best-performance. Accessed 21 Nov 2019

22. Devineau G, Xi W, Moutarde F et al. (2018) Convolutional Neural Networks for Multivariate Time Series Classifcation using both Inter-\& Intra-Channel Parallel Convolutions. https://rfiap2018.ign.fr/sites/default/files/ARTI CLES/RFIAP_2018/RFIAP_2018_Devineau_C onvolutional.pdf

23. Groß W, Lange S, Bödecker $\mathrm{J}$ et al. Predicting Time Series with Space-Time Convolutional and Recurrent Neural Networks. European Symposium on Artificial Neural Networks, Computational Intelligence and Machine Learning 2017

24. Greff D (2019) Investigating the development of a dynamic weighing system for forestry machines: -using a data driven approach-. Master's thesis, Karlsruher Institut für Technologie

25. Hohenlohe, Felix, zu, Geiger C, Geimer M (2019) Hybridantrieb für den Ladekran einer Forstmaschine. In: Hybride und energieeffiziente Antriebe für mobile Arbeitsmaschinen : 7. Fachtagung. KIT Scientific Publishing, Karlsruhe

26. Nair V, Hinton GE (2010) Rectified linear units improve restricted boltzmann machines. ICML'10 Proceedings of the 27th International Conference on International Conference on Machine Learning: 807-814

27. Goodfellow I, Bengio Y, Courville A (2016) Deep Learning. MIT Press

28. Hastie T, Tibshirani R, Friedman JH (eds) (2017) The elements of statistical learning: Data mining, inference, and prediction, Second edition, corrected at 12th printing 2017. Springer series in statistics. Springer, New York, NY

29. Deutschen Forstwirtschaftsrates e.V., Deutschen Holzwirtschaftsrates e.V. (2015) Rahmenvereinbarung für den Rohholzhandel in Deutschland (RVR) 